\title{
Effects of study task on the neural correlates of source encoding
}

\author{
Heekyeong Park, ${ }^{1,2,3}$ Melina R. Uncapher, ${ }^{1,2}$ and Michael D. Rugg ${ }^{1,2}$ \\ ${ }^{1}$ Center for the Neurobiology of Learning and Memory, University of California, Irvine, California 92697-3800, USA; ${ }^{2}$ Department \\ of Neurobiology and Behavior, University of California, Irvine, California 92697-3800, USA
}

\begin{abstract}
The present study investigated whether the neural correlates of source memory vary according to study task. Subjects studied visually presented words in one of two background contexts. In each test, subjects made old/new recognition and source memory judgments. In one study test cycle, study words were subjected to animacy judgments, whereas in another cycle the study task required syllable judgments. Functional magnetic resonance imaging (fMRI) was employed to contrast the neural activity elicited by study words that attracted accurate source judgments on the later memory test, as opposed to words for which source judgments were incorrect or for which source information was unavailable. In both tasks, relative to words for which source memory failed, study words that were later assigned to the correct source elicited enhanced activity in ventral extrastriate cortex. In addition to these common effects of subsequent source memory, additional effects were observed that were selective for each study task. The present findings add weight to the proposal that neural activity supporting successful episodic memory encoding is a reflection of both the online processing engaged by an episode as it is experienced, and the demands imposed by the later retrieval task.
\end{abstract}

Numerous studies of memory encoding have reported that, as indexed by functional magnetic resonance imaging (fMRI), the neural activity elicited by a study item differs according to whether the item is remembered or forgotten on a later memory test (for early studies see Brewer et al. 1998; Wagner et al. 1998; Henson et al. 1999; Kirchhoff et al. 2000; for reviews, see Paller and Wagner 2002; Davachi 2006). These differences in study activity are often termed "subsequent memory effects." They have been described in a variety of different cortical regions, as well as in the hippocampus and adjacent regions of the medial temporal lobe.

Subsequent memory effects are frequently interpreted as reflections of the cognitive and neural processes that support successful memory encoding, but their functional significance remains unclear. Importantly, they have been reported to dissociate depending on whether memory for study items is associated with retrieval of contextual details about the study episode ("recollection"), or is based on an acontextual sense of familiarity (Davachi et al. 2003; Ranganath et al. 2004; Uncapher and Rugg 2005). Such dissociations add credence to "dual-process" models of memory (Yonelinas 2002), and indicate that episodic memory depends upon encoding operations different from, or in addition to, those that support item-specific memory.

An important clue to the functional significance of subsequent memory effects comes from findings that their cortical loci are sensitive to study task manipulations (Otten and Rugg 2001; Otten et al. 2002; Fletcher et al. 2003; Mitchell et al. 2004). For example, in the studies of Otten and Rugg (2001) and Otten et al. (2002) study words were classified either in terms of their semantic (animate/nonanimate) or phonological (odd/even number of syllables) characteristics. Across the two experiments, subsequent memory effects demonstrated regional double-dissociations according to study task: The effects associated with the semantic

${ }^{3}$ Corresponding author.

E-mail hkpark@uci.edu; fax (949) 824-4807.

Article is online at http://www.learnmem.org/cgi/doi/10.1101//m.878908. task overlapped in part with regions implicated in the performance of online semantic judgments (e.g., left inferior frontal gyrus and medial prefrontal cortex), whereas the loci of the effects for the syllable task included regions that are also active during syllabic judgments (e.g., bilateral parietal and fusiform cortex). These results have been construed (e.g., Rugg et al. 2002, 2008 ) as evidence against the proposal that episodic memory encoding depends upon a single cortical region or network (e.g., Habib et al. 2003), and as support for the idea that memories are encoded in terms of the cognitive operations engaged during the online processing of a study item (e.g., Morris et al. 1977; Roediger et al. 2002).

The findings described above demonstrate that the cortical correlates of successful long-term memory encoding differ depending on the processing accorded study items, but their significance for the understanding specifically of episodic memory is uncertain. This is because no study has yet investigated the influence of study task on subsequent memory effects with memory tests that segregate later remembered items according to whether or not memory was based on recollection. Thus, while prior findings strongly suggest that the cortical loci of the encoding of study items vary according to study task, this has not been demonstrated for the encoding of study episodes (i.e., item plus context). Claims that the cortical correlates of episodic encoding are task-dependent (e.g., Rugg et al. 2002, 2008) are therefore open to question.

As already noted, a defining feature of episodic memory is retrieval of contextual information associated with a study item (i.e., accurate source memory) (Johnson et al. 1993). Several fMRI studies have employed source memory tests along with the subsequent memory procedure to investigate the neural correlates of successful versus unsuccessful source encoding (Cansino et al. 2002; Davachi et al. 2003; Ranganath et al. 2004; Gold et al. 2006; Kensinger and Schacter 2006; Uncapher et al. 2006; Tendolkar et al. 2007). To our knowledge, however, no study has contrasted subsequent source memory effects as a function of study task. 
The goal of the present study was to investigate whether subsequent memory effects are modulated by study task when the later memory test requires retrieval of information about both item and source (context) information. Study words were presented in one of two physically distinct display contexts. In one study test cycle, the words were subjected to a syllabic judgment, whereas in the other cycle the study task involved animacy judgments. In both test phases, the requirement was to identify the studied words and to indicate the context in which they had been presented. If the encoding operations supporting episodic memory (as operationalized by accurate source judgments) are task-dependent, then the associated subsequent memory effects (derived from the contrast between study activity elicited by later recognized items accorded accurate vs. inaccurate source judgments) will demonstrate dissociations similar to those reported by Otten et al. (2001) and Otten and Rugg (2001). Furthermore, these task-specific effects should overlap, at least in part, with regions selectively activated by the respective study tasks (Otten and Rugg 2001). By contrast, if the task-sensitive subsequent memory effects described in those studies reflect encoding operations that support item-specific memory, they will not be manifest in subsequent memory effects associated with successful source memory, but will be evident in the effects associated with successful item memory (the contrast between study activity elicited by recognized items accorded incorrect source judgments vs. forgotten items).

In addition, the question also arises whether any encoding processes supporting successful source memory are engaged independently of the specific study task. In particular, to the extent that the hippocampus and adjacent medial temporal cortex are necessary for the formation of item-context associations (Davachi 2006; Eichenbaum et al. 2007), items associated with later successful source judgments should elicit medial temporal subsequent memory effects regardless of the study task.

\section{Results}

\section{Behavioral results}

\section{Study phase}

Animacy judgments were made with an accuracy of 95\% (SD = 3) and a mean reaction time (RT) of $1253 \mathrm{~ms}(\mathrm{SD}=262)$; accuracy for syllable judgments was also $95 \%(\mathrm{SD}=4)$, but with a mean RT of $1428 \mathrm{~ms}(\mathrm{SD}=332)$. Unsurprisingly, accuracy did not differ significantly according to type of study task, but RTs were significantly longer for syllable judgments than for animacy judgments $\left(F_{(1,16)}=15.69, p<0.005\right)$. RTs segregated according to later memory performance are shown in Table 1 . To parallel the approach taken in the fMRI analyses (see below), RTs were contrasted according to whether later recognized items were accorded correct source judgments (source hit) as opposed to attracting either incorrect or unsure judgments. The RTs for these classes of study items did not differ significantly, nor was there any evidence for an interaction between subsequent memory and study tasks.

Table 1. Mean study reaction times (ms) according to subsequent memory judgments (SD in parentheses)

\begin{tabular}{lcccc}
\hline & \multicolumn{3}{c}{ Old } & \\
\cline { 2 - 4 } & $\begin{array}{c}\text { Source } \\
\text { correct }\end{array}$ & $\begin{array}{c}\text { Source } \\
\text { incorrect }\end{array}$ & $\begin{array}{c}\text { Source } \\
\text { unsure }\end{array}$ & New \\
\hline $\begin{array}{l}\text { Animacy } \\
\text { Syllable }\end{array}$ & $1256(275)$ & $1234(239)$ & $1239(264)$ & $1223(243)$ \\
\hline
\end{tabular}

Table 2. Mean proportions of source judgments for correctly recognized study items (SD in parentheses)

\begin{tabular}{lccc}
\hline & Source correct & Source incorrect & Source unsure $^{\text {a }}$ \\
\hline Animacy & $0.58(0.13)$ & $0.16(0.10)$ & $0.26(0.13)$ \\
Syllable & $0.45(0.13)$ & $0.20(0.13)$ & $0.35(0.17)$ \\
\hline
\end{tabular}

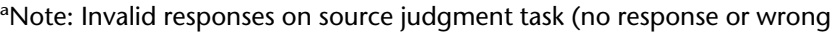
button press) were assigned as source unsure responses.

\section{Test phase}

Mean hit rates (collapsed over source accuracy) for studied items on the later memory test were $0.77(\mathrm{SD}=0.14)$ in the animacy task and $0.66(\mathrm{SD}=0.14)$ in the syllable task, with false alarm rates of $0.08(\mathrm{SD}=0.05)$ and $0.13(\mathrm{SD}=0.13)$, respectively. The discrimination index, $\operatorname{Pr}\left(p_{\text {hit }}-p_{\mathrm{FA}}\right)$ (Snodgrass and Corwin 1988) indicated that words studied in the animacy task were more likely to be recognized than words from the syllable task $\left(F_{(1,16)}=18.2, p<0.005\right)$. As is evident from Table 2 , words recognized from the animacy task were also more likely to be accorded accurate source judgments (source hits) than were words from the syllable task. To correct for the influence of guesses, source hit rates were adjusted according to a single high threshold model, using the formula, $p_{\text {corrected source hit }}=\left[p_{\text {source hit }}-\right.$ $\left.0.5\left(1-p_{\text {source unsure }}\right)\right] /\left[1-0.5\left(1-p_{\text {source unsure }}\right)\right]$. The adjusted source hit rates were $0.34(\mathrm{SD}=0.15)$ and $0.18(\mathrm{SD}=0.15)$ for the animacy and syllable tasks, respectively, and these differed significantly $\left(F_{(1,16)}=23.18, p<0.001\right)$. There was no effect on the order in which the study tasks were administered on the accuracy of source judgments, nor was there any evidence of an order $\times$ task interaction.

\section{fMRI results}

\section{Analysis overview}

The subsequent source memory analyses described below are based on contrasts between the activity elicited by study items that were both correctly recognized and attributed to the correct source (source hit) versus the activity elicited by items that were recognized but for which the source judgment was either inaccurate or unsure. This contrast isolates activity selectively associated with successful source retrieval while holding item memory constant. Contrasts between study activity associated with successful versus unsuccessful item memory, that is, activity elicited by items accorded source incorrect or source unsure judgments versus misses, are reported only briefly, since five subjects had too few miss trials $(<12)$ in the animacy task to allow estimation of stable parameter estimates.

\section{Task-invariant subsequent source memory effects}

First, we sought to identify subsequent source memory effects that were shared between the two study tasks. We accomplished this by first computing the main effect of subsequent source memory (i.e., source hit vs. source incorrect + source unsure), peak thresholded at $p<0.005$ with a cluster extent threshold of $p<0.05$. To include only voxels where subsequent memory effects were independently significant in each task, the main effect was then inclusively masked with both simple effects, each thresholded at $p<0.05$. Finally, the contrast was exclusively masked by the interaction ( $F$ contrast) between study task and subsequent memory. This mask was thresholded at $p<0.1$, giving a threshold for each side of the interaction of $p<0.05$. The outcome of this analysis is illustrated in Figure 1A, where it can be seen that the region demonstrating common effects extends across the left ventral occipital cortex. Parameter estimates for 
A
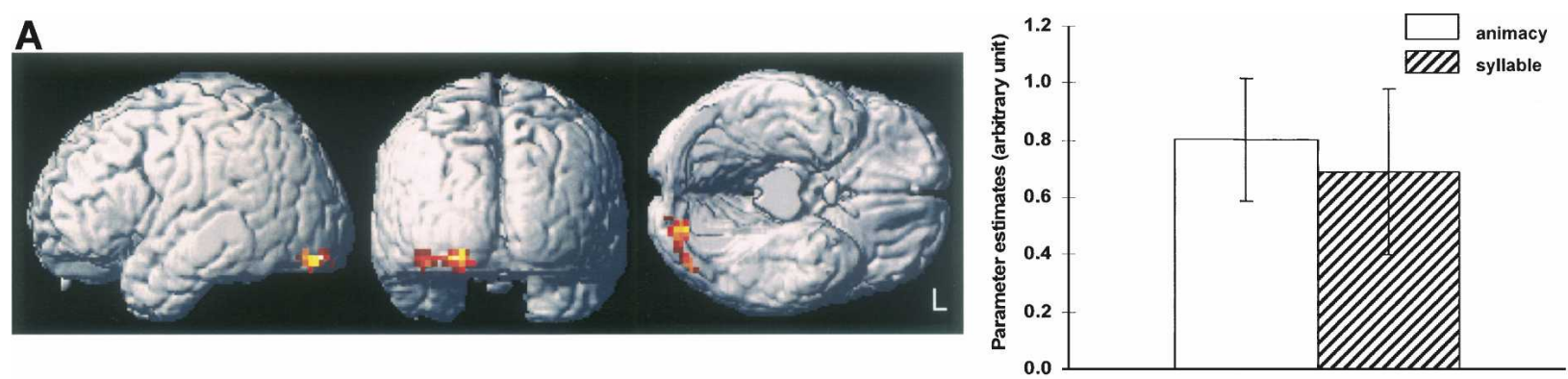

B

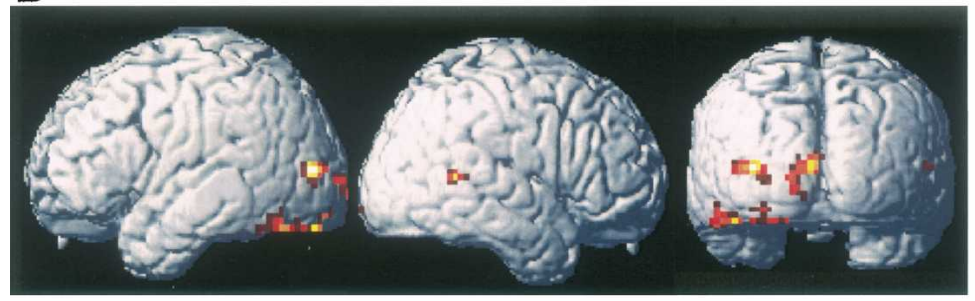

C
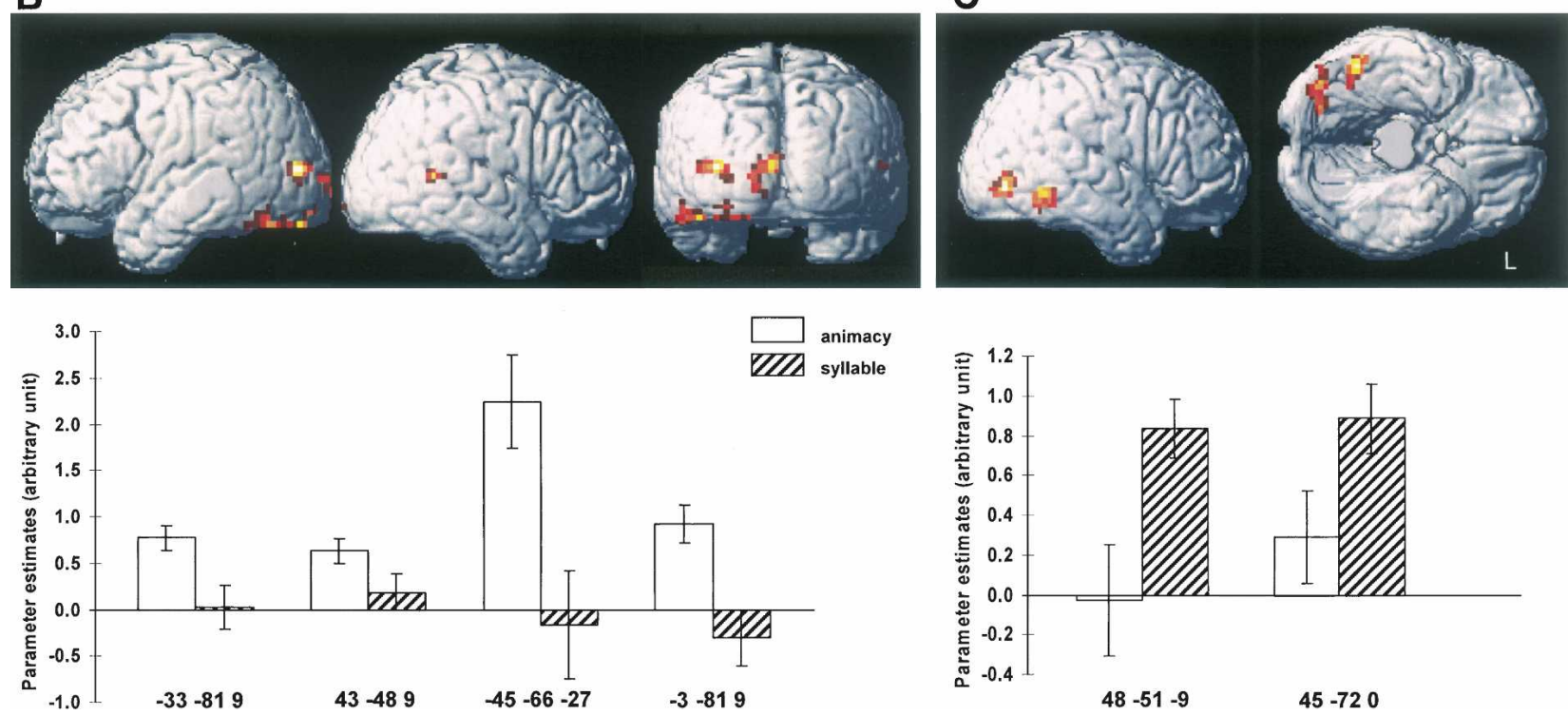

syllable

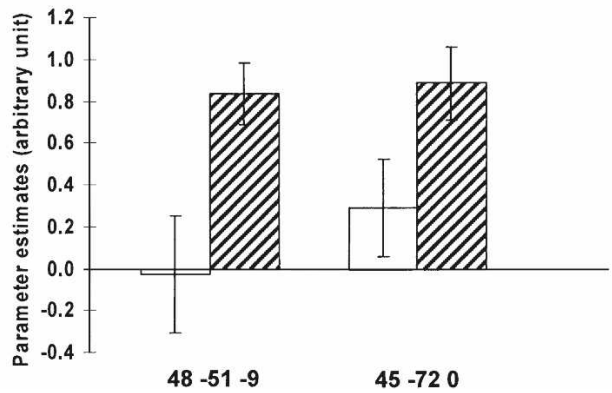

Figure 1. (A) Task-invariant subsequent source memory effects $(p<0.005)$, overlaid on a rendered canonical normalized brain (with the cerebellum artificially removed). Graph depicts the mean and standard error of the parameter estimates of the subsequent source memory effects associated with each study task. $(B)$ Task-specific subsequent source memory effects $(p<0.005)$ and associated peak parameter estimates for the animacy study task. (C) Task-specific subsequent source memory effects and parameter estimates for the syllable task.

the peak voxels of these subsequent memory effects are also shown in Figure 1A.

\section{Task-specific subsequent source memory effects}

The second part of the analysis identified regions where subsequent source memory effects were unique to one or the other study task. This was accomplished by exclusively masking the subsequent memory contrast $(p<0.005$, cluster extent $p<0.05)$ for the study task of interest (e.g., animacy source hit vs. animacy source incorrect + source unsure), with the contrast for the alternate task, peak thresholded at $p<0.05$. The outcomes of these analyses are listed in Table 3 and illustrated, along with peak parameter estimates, in Figure 1B (animacy-specific effects) and Figure 1C (syllable-specific effects). Regions uniquely associated with successful encoding in the animacy task include the left extrastriate and the left fusiform cortex, and the right temporalparietal junction. Subsequent memory effects associated specifically with the syllable task were found in the right inferior occipital and temporal cortex.

Statistical dissociation between task-invariant and task-specific subsequent source memory effects

The foregoing analyses employed a combination of inclusive and exclusive masking to identify regions where subsequent source memory effects were either of equivalent magnitude in the two encoding tasks, or were exclusive to one or the other task. These analyses leave open the question whether the different patterns of effect are statistically dissociable. To address this question, it is necessary to contrast the subsequent source memory effects arising from regions identified as exhibiting task-invariant versus task-specific effects. To perform this analysis we first averaged the

Table 3. Regions showing task-specific subsequent source memory effects

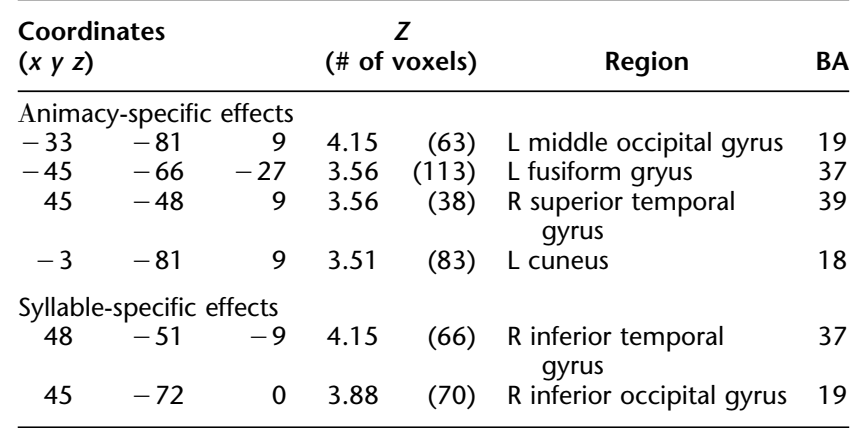

Coordinates and $Z$-values refer to the peak voxels of activated clusters. $L$, left; $R$, right; BA, Brodmann area (approximate). 
peak parameter estimates for the subsequent memory effects from the clusters demonstrating each type of effect and then performed a two-way ANOVA with factors of effect type (invariant, animacy specific, and syllable specific) and task (animacy, syllable). This gave rise to a significant effect type $\times$ task interaction $\left(F_{(1.5,23.8)}=31.17, p<0.001\right.$; the degrees of freedom are reported after Greenhouse-Geisser correction for nonsphericity). Follow-up ANOVAs separately contrasting the task-invariant effects with each class of specific effects also gave rise to effect type $\times$ task interactions (animacy task: $F_{(1,16)}=5.64, p<0.05$; syllable task: $\left.F_{(1,16)}=21.19, p<0.001\right)$. These findings indicate that the taskinvariant and task-specific effects do indeed reflect qualitatively distinct patterns of neural modulation.

\section{Subsequent source memory effects in the medial temporal lobe}

In light of prior reports of the involvement of the hippocampus and adjacent medial temporal cortex in the encoding of items attracting later successful source judgments (e.g., Davachi et al. 2003; Ranganath et al. 2004; Kensinger and Schacter 2006; Uncapher et al. 2006), we searched for evidence of task-invariant and task-specific medial temporal subsequent memory effects using a relaxed statistical threshold for the principal contrasts. No clusters were revealed in the medial temporal lobe for either task-invariant or syllable-specific subsequent source memory contrasts, even when the threshold was reduced to $p<0.05$. For the animacy-selective contrast, however, reducing the peak threshold to $p<0.01$ revealed a subsequent source memory effect in the right hippocampus (peak at $30,-15,-12$, $Z=2.86, p<0.005$, cluster size $=15$; see Fig. $2 \mathrm{~A}$ ). The effect was very close to the hippocampal region reported by Uncapher et al. (2006) as demonstrating a subsequent memory effect for successful source memory (peak at $27,-15,-15$ ), and survived a small volume correction $(p<0.05)$ for a 5 -mm sphere centered on the coordinates of the previously reported effect. As is evident from Figure $2 \mathrm{~A}$, there was no hint of a subsequent source memory effect in this region for the syllable task. The ANOVA of the respective parameter estimates gave rise to a significant task effect $\left(F_{(1,16)}=5.60, p<0.05\right)$.

\section{Overlap between subsequent source memory and task effects}

Following Otten and Rugg (2001) we searched for regions demonstrating overlap between task-specific subsequent source memory and task effects by inclusively masking each subsequent source memory effect by the corresponding task contrast. To identify overlap between animacy subsequent source memory effects and regions selectively activated by the animacy task, we masked the contrast between animacy study items attracting correct versus incorrect/unsure source judgments $(p<0.005$, exclusively masked by the corresponding contrast for the syllable task, $p<0.05$ ) by the contrast between activity elicited by all study items in the animacy versus syllable task $(p<0.005$, giving a conjoint significance for these two contrasts of $p<0.0003$ as estimated by Fisher's procedure) (Lazar et al. 2002). The analogous procedure was followed for the syllable effects. In the case of the syllable task, a cluster of 57 voxels was identified in the right lateral occipital cortex (peak at $39,-75,-6, Z=3.87$ ). No regions of overlap were identified, however, for the animacy task. Lowering the thresholds for the respective contrasts to $p<0.05$ revealed only a few scattered clusters, none greater than five voxels.

\section{Subsequent item memory effects}

Task-invariant and task-specific subsequent item memory effects were identified using the same procedures as were employed for the source memory effects, this time focusing on contrasts between study items that attracted incorrect or unsure source judgments versus items that were missed on the later test. As noted previously, these analyses were performed on only a subset $(n=12)$ of the subjects included in the analysis of subsequent source effects.

Task-invariant subsequent item memory effects were evident in two regions, the right ventral occipital (BA 18, peak at 3, $-72,-9, Z=3.92,102$ voxels) and the right medial prefrontal cortex (BA 8; peak at 3, 30, 48, $Z=3.71,41$ voxels). Task-specific item effects for the animacy task were confined to the left superior prefrontal region (BA 8; peak at $-24,45,42, Z=3.16,44$ voxels). In the case of the syllable task, specific effects were evident in, among other regions, the bilateral insula and the anterior medial temporal lobe including the hippocampal formation (see Fig. 2B). Of most relevance in relation to prior findings, task-specific syllable effects were also present in the left lateral prefrontal cortex (BA 9, peak $-45,21,33, Z=3.67,85$ voxels). This and the analogous animacy effect are illustrated in Figure 3. An ANOVA (factors of effect type and task) of the peak parameter estimates from these two prefrontal regions gave rise to a significant interaction $\left(F_{(1,11)}=14.66, p<0.005\right)$, indicative of a dissociation between the patterns of subsequent item memory effect in the two regions. 

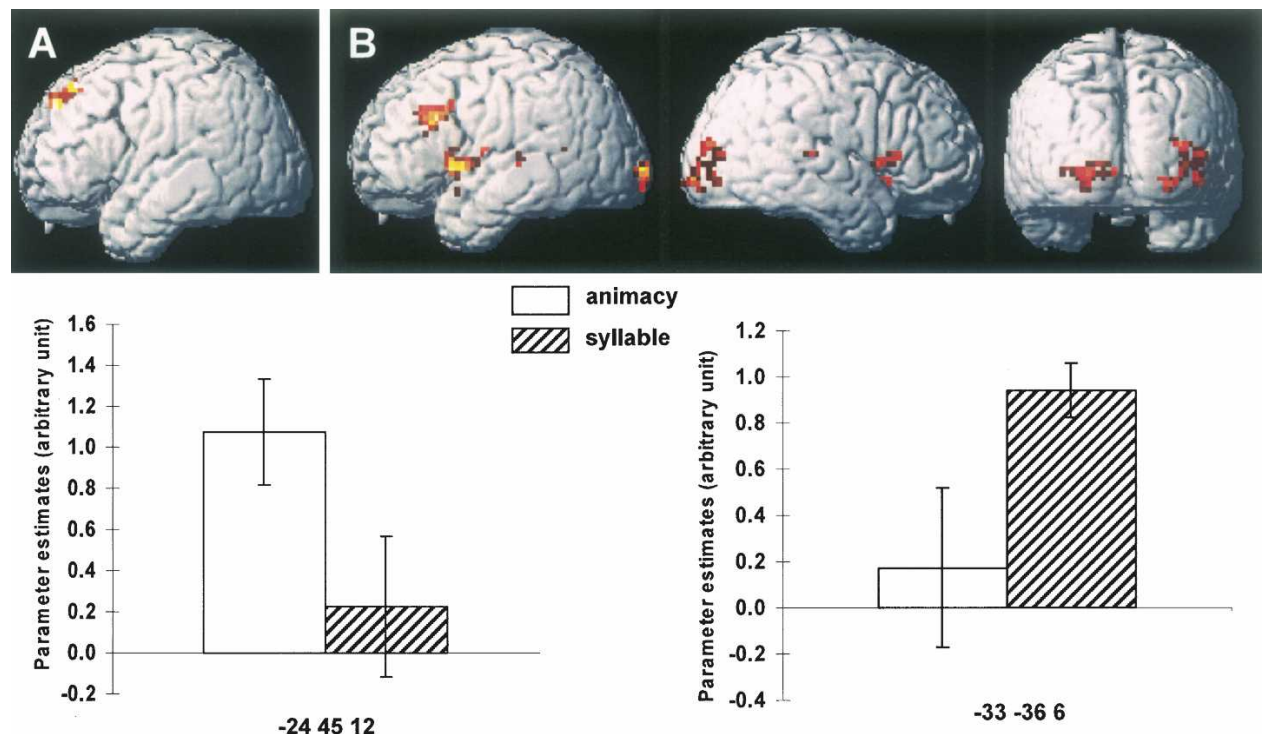

animacy

$-244512$

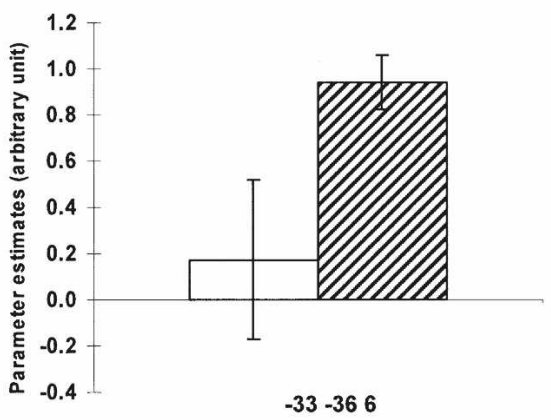

Figure 3. Task-specific subsequent item memory effects. $(A)$ Animacy-specific item memory effects and parameter estimates. (B) Syllable-specific item memory effects and parameter estimates. Effects are threshold at $p<0.005$ and overlaid on a rendered canonical normalized brain (with the cerebellum artificially removed). Error bars indicate the standard error of the mean.

\section{Discussion}

\section{Behavioral findings}

As in previous studies, animacy judgments were made $\sim 200 \mathrm{~ms}$ more quickly than syllable judgments, although with equivalent accuracy. In neither task were there detectable RT differences between study items according to later memory performance. Thus, whereas the difference between the tasks in overall response latency must be borne in mind when interpreting taskdependent fMRI subsequent memory effects, the effects themselves are unlikely to reflect gross inequities in the efficiency with which different study items were processed.

The present behavioral findings replicate many previous results in demonstrating the expected advantage for semantic over nonsemantic study on recognition memory. The animacy task also gave rise to higher levels of source accuracy, consistent with prior reports that experimental manipulations that influence item memory typically exert a parallel influence on source memory (Bornstein and LeCompte 1995; Hayman and Rickards 1995; Glanzer et al. 2004), at least in young adults (NavehBenjamin and Craik 1996). In a conventional forced choice source memory task, differential levels of source accuracy would imply differences in the proportion of accurate source judgments contributed by lucky guesses (the lower the performance, the greater the proportion of guesses). In turn, this would lead to the expectation of weaker neural correlates of successful source memory in the condition associated with the poorer performance, as a result of the smaller proportion of trials associated with veridical recollection. It is unlikely, however, that differential guessing effects played a significant role in the present case. Unlike in most prior studies, the source memory test employed here included a response option that allowed subjects to signal when they were unsure of the source of a recognized item. By including this option we aimed to reduce the proportion of accurate source judgments that were due to lucky guesses rather than veridical recollection. As is evident from Table 2, the "unsure" response option served its purpose well; it was employed for more than a quarter of recognized items studied in the animacy task, and over a third of the items recognized from the syllable task.

\section{fMRI results}

The principal aim of this study was to determine whether the neural correlates of successful source encoding vary according to study task. Subsequent source memory effects common to the two study tasks were evident in one region, but there were other regions where the effects were specific to one task or the other. Below, we discuss the implications of these and related findings.

\section{Task-invariant source memory effects}

The finding of task-invariant effects suggests that the successful encoding of a study item along with its context depended, in part, on processes that were shared across the two tasks. The localization of these effects to the ventral extrastriate visual cortex suggests that later source memory was facilitated on trials where early visual processing was emphasized. Since the contextual features associated with the study items were the visual attributes of location and color, this is perhaps unsurprising. Indeed, the task-invariant subsequent memory effect overlaps with the area of the human V4 complex that has been held to play a key role in color processing (Zeki and Marini 1998; Bartels and Zeki 2000), suggesting that color information may have been especially important in supporting later source judgments. This finding adds further weight to the proposal that there is a close relationship between the resources allocated to the online processing of a particular attribute of an episode, and the likelihood that the attribute will form part of the ensuing memory representation (e.g., Rugg et al. 2002, 2008; Uncapher et al. 2006). More tentatively, the present findings arguably also add to recent evidence that the neural correlates of successful encoding depend on the nature of both the study and the retrieval task (Otten 2007; Park and Rugg 2008). By this argument, although retrieval of task-specific information would suffice to support later recognition of the study items, the information supporting accurate source memory judgments was common to the two tasks; thus, for both study tasks, the later source memory test "selected for" study trials on which processing of source-specifying information received emphasis. According to this account, task-invariant subsequent memory effects should be evident whenever the later memory test requires retrieval of information common to the different classes of study episode. 


\section{Task-specific subsequent source memory effects}

In addition to the task-invariant subsequent source memory effects discussed above, robust task-specific effects were also evident. In the cortex, these effects took the form of double-dissociations between posterior regions where subsequent memory effects were manifest exclusively for the animacy or the syllable task.

We assume that the task-specific effects reflect modulation of processes selectively engaged by the respective study tasks. Thus, one of the regions demonstrating an animacy-specific effect-the left posterior fusiform cortex-is close to regions where subsequent memory effects have been identified in several previous studies that employed semantically oriented study tasks (e.g., Wagner et al. 1998; Kirchhoff et al. 2000; Otten and Rugg 2001; Morcom et al. 2003). The animacy task was also associated with subsequent source memory effects in the medial and lateral occipital cortex (BA 18/19), regions that have also been identified in prior studies employing the same encoding task (Otten et al. 2001; Morcom et al. 2003). An animacy-selective effect was also evident in the right temporo-parietal cortex. To our knowledge, however, subsequent memory effects for semantically mediated encoding have not previously been reported in this region.

Subsequent source memory effects selective for the syllable task were found in the right occipital and posterior fusiform cortex. These clusters are in the vicinity of the lateral occipital and posterior fusiform subdivisions of the lateral occipital complex (Lerner et al. 2002), a region held to play a role in the early stages of object recognition (Grill-Spector et al. 2001). Subsequent memory effects associated with syllabic encoding were reported in an area near the more anterior of these regions by Otten and Rugg (2001) $(48,-48,-21)$, and in a near-homotopic region by Otten et al. (2002) $(-42,-60,-9)$. Together with these previous findings, the present results appear to implicate the posterior fusiform in the episodic encoding of items subjected to syllable judgments. In light of the overlap of this region with areas implicated in visual object processing, it is tempting to speculate that the region contributes to the encoding of a visually based memory representation of the study items; the form of this representation, and the role it might play in the support of online syllable judgments, are obscure.

Otten and Rugg (2001) reported that a subset of their animacy- and syllable-specific subsequent item memory effects overlapped with regions where activity was selectively enhanced for the corresponding task. This finding was interpreted as evidence that subsequent memory effects localize to cortical regions engaged during online processing of the study episode (see also Mitchell et al. 2004; Uncapher et al. 2006; Rugg et al. 2008). Consistent with this proposal, and our pre-experimental prediction (see Introduction), the right occipital, syllable-specific subsequent source memory effect in the present study overlapped with a region demonstrating greater study item activity in the syllable than in the animacy task. No regions of overlap were evident, however, between animacy-selective subsequent source memory effects and the corresponding task effect. This null finding should be treated with caution, however, since the task contrast (animacy > syllable) by itself revealed very weak effects (only three midline clusters, varying between 10 and 29 voxels in size, for $p<0.005$ and a nine voxel extent threshold). These weak effects may be a consequence of the relative lack of statistical efficiency associated with between- rather than within-session contrasts (Smith et al. 2005).

\section{Subsequent item memory effects}

As noted already, caution is necessary when interpreting the outcome of the analyses of subsequent item memory effects, as these were based on only a subset of the subjects contributing data to the analyses of source memory effects. Nonetheless, it is noteworthy that these analyses also revealed a regional doubledissociation between subsequent memory effects in each task. Most notably, the effects included two prefrontal regions that are close to regions reported as showing animacy- or syllable-specific subsequent memory effects in prior studies (Otten and Rugg 2001; Otten et al. 2001, respectively). Together with the analyses of the subsequent source memory effects discussed above, these findings suggest that the task-specific subsequent memory effects described in those prior studies reflected an amalgam of processes supporting the encoding of item information and item-context associations.

\section{Hippocampal subsequent memory effects}

Hippocampal subsequent memory effects have been reported specifically for study items attracting correct versus incorrect source judgments (Davachi et al. 2003; Ranganath et al. 2004; Kensinger and Schacter 2006; Uncapher et al. 2006) as well as more generally (e.g., Otten et al. 2001; Fletcher et al. 2003; Morcom et al. 2003; Uncapher and Rugg 2005; Gold et al. 2006). In the present case, hippocampal subsequent source memory effects were found for the animacy task in a region very close to that reported by Uncapher et al. (2006) in a study that also assessed source memory for color and location. This finding is consistent with proposals that the hippocampus plays a key role in the formation of item-context associations (e.g., Davachi 2006; Eichenbaum et al. 2007). The failure to find analogous effects in the syllable task might suggest that, like some cortical regions, encoding operations in the hippocampus are sensitive to whether the study episode engages semantic processing. Interpretation of this null finding is complicated, however, by the marked disparity in source memory performance between the two tasks. Thus, the absent syllable subsequent source memory effects may simply be a reflection of the relative paucity of source-specifying information encoded in the syllable task, even for study trials that later attracted correct source judgments. By this argument, hippocampal subsequent source memory effects were absent in the syllable task because the amount of contextual information encoded in trials associated with successful versus unsuccessful source judgments did not differ sufficiently to modulate hippocampal activity to a detectable extent, and not because the hippocampus responds in a qualitatively different manner during semantic versus nonsemantic study tasks.

More compelling evidence that hippocampal subsequent memory effects are not limited to semantically oriented study tasks comes from the contrasts that identified effects associated with later successful item memory. In the syllable task only, robust subsequent item memory effects were evident in the bilateral medial temporal lobe, including the hippocampus (see Fig. 2B). It is unclear why hippocampal subsequent memory effects distinguished between successful and unsuccessful source memory in the animacy task, but between successful and unsuccessful item memory in the syllable task. One possibility is that item memory in the syllable task was more likely to be associated with noncriterial recollection (i.e., recollection of contextual features that were nondiagnostic of source) (Yonelinas and Jacoby 1996) than in the animacy task.

\section{Conclusions}

The present study demonstrates that the neural correlates of successful source memory encoding vary according to study task. The findings thus represent an important extension to the results of prior studies (Otten and Rugg 2001; Otten et al. 2001), indicating that the cortical loci of the encoding not only of specific study items, but of study episodes (item-context association) depend upon the processing engaged during study. The findings 
are consistent with the principle, long articulated in psychological theories of memory (e.g., Kolers 1973; Morris et al. 1977), that episodic memory is a by-product of the online processing accorded an episode as it is experienced.

\section{Materials and Methods}

\section{Subjects}

Seventeen subjects participated in the experiment (aged 18-20 yr; seven males). They were recruited from the University of California, Irvine (UCI) undergraduate community and were compensated for their participation. An additional three subjects were excluded from analysis due to excessive head movement or falling asleep during scanning. All subjects were right-handed, native English speakers, and gave informed consent prior to their participation. The experiment was approved by the UCI Institutional Review Board.

\section{Materials}

The stimulus materials were drawn from a word pool of 360 nouns (4-9 letters, in the range of 1-30 word frequency counts per million) (Kučera and Francis 1967). Three sets of 120 words were constructed from the word pool. Half of each set contained animate items, and half inanimate items. In addition, half of the words contained an odd number of syllables and half an even number. For each subject two study lists, one for the animacy task and the other for the syllable task, were created from these word sets by random selection of items without replacement. Each study list was composed of 96 critical words with an additional four buffer items. There were two test lists, each consisting of 146 words. These were composed of a random sequence of 96 studied words from the corresponding study list, 48 new words, and an additional two buffer items. The words from the original word pool not selected as study or test items were used for study and test practice trials.

Words were displayed with VisuaStim (Resonance Technology, Inc.) XGA MRI compatible head-mounted goggles with a resolution of $800 \times 600$ pixels. The field of display subtended visual angles of $30^{\circ} \times 23^{\circ}$ at a virtual viewing distance of $1.2 \mathrm{~m}$. During study, the critical words were presented either in an orange frame on the left side or in a purple frame on the right side of the visual field, preceded by a central task cue ("o" for the animacy task, " $\mathrm{x}$ " for the syllable task). Each frame subtended visual angles of $8.4^{\circ} \times 8.4^{\circ}$. The centers of the frames were displaced $8.5^{\circ}$ to the left or right of fixation, respectively. Words were presented in white upper case Helvetica 30 point font, at a maximum visual angle of $8^{\circ} \times 1.5^{\circ}$. During the test phase, words were presented in the same size and font as at study, but at the center of the visual field against a gray, $8.4^{\circ} \times 8.4^{\circ}$ box.

\section{Procedure}

Subjects were given instructions and practice outside the scanner on both the study and test tasks prior to the experiment proper. There were two study test cycles, one for the animacy task, and the other for the syllable task. Each study and test phase comprised a separate scanning session. The order of the tasks was counterbalanced over subjects. For each study phase, the task cue (an o or $\mathrm{x}$, depending on the task; this served to alert subjects to the upcoming item, and to instruct them which task they should perform) was presented for $600 \mathrm{~ms}$, followed immediately by the study word, which was displayed for $1000 \mathrm{~ms}$. The word was replaced by a white fixation asterisk for $1800 \mathrm{~ms}$ prior to the onset of the next task cue. Stimulus onset asynchrony was thus $3400 \mathrm{~ms}$. Each list contained 48 null trials, during which a fixation character (+) was continuously displayed for $3400 \mathrm{~ms}$ and no response was required. The presentation order of study words in a list was pseudorandom, with the constraint that there were no more than three consecutive trials of one item type, including null trials.

For the animacy study task, subjects were instructed to make an animate/inanimate judgment for each word and to press a corresponding button with either the index or middle finger of their right hand. The assignment of finger to the animate/ inanimate response was counterbalanced across subjects. Half of the critical study words were presented in the left/orange frame and the other half were presented in the right/purple frame in a pseudorandom order. No more than three consecutive study items were presented in the same location. Subjects were reminded about the nature of the subsequent memory tests prior to the start of the study phase. For the syllable study task, subjects were required to judge whether the number of syllables of each study word was odd or even, and to indicate their response with either the index or middle finger of their right hand. All other display parameters and task instructions were as for the animacy task.

The test phase followed $\sim 5$ min after the end of the study task. Test procedures were the same for both study tasks. All stimuli were presented at fixation superimposed upon a gray background. Each test trial consisted of the presentation of a fixation cross (+) for $100 \mathrm{~ms}$, followed by the presentation of the test word for $600 \mathrm{~ms}$. The word was followed by an asterisk that was displayed for $2000 \mathrm{~ms}$. Subjects were required to judge whether or not they had seen the word in the immediately preceding study session, and to indicate their decision by button press using the index or middle finger of the right hand (the finger assigned to each response was counterbalanced across subjects). For items endorsed as "old," subjects were then required to judge whether the item had been presented at study on the left or the right of the display monitor. The source judgment was prompted by presentation of the word "Location," under which were presented the three response options (left, right, and unsure). These responses were made using the ring, middle, and index fingers of the left hand. For items endorsed as "new," no further response was required.

\section{fMRI scanning parameters}

A 1.5 Philips Eclipse MR (Philips Medical Systems) scanner was used to acquire both T1-weighted anatomical volume images $\left(256 \times 256\right.$ matrix, $1 \mathrm{~mm}^{3}$ voxels $)$ and $\mathrm{T} 2 *$-weighted gradient echo-planar images $(64 \times 92$ matrix, $2.6 \times 3.9 \mathrm{~mm}$ pixels, $\mathrm{TE}=40 \mathrm{~ms}$ ) per volume with blood oxygenation level dependent (BOLD) contrast. EPIs were composed of 25 axial slices that were $3 \mathrm{~mm}$ thick with a $2 \mathrm{~mm}$ interslice gap, acquired in descending sequential order. Scanning took place during the study and test phases. Scanning for the study phases involved in each case the acquisition of 240 volumes at a repetition time (TR) of $2400 \mathrm{~ms}$. The first five volumes of each session were discarded to allow tissue equilibration. The ratio of SOA to TR in the study phase resulted in a sampling of the impulse response at a rate of $\sim 2 \mathrm{~Hz}$ over the trials. A T1-weighted anatomical volume covering the whole brain was acquired for each subject after subjects completed the two study test cycles.

\section{Data analysis}

Statistical Parametric Mapping (SPM2; Wellcome Department of Cognitive Neurology: http://www.fil.ion.ucl.ac.uk/spm) was used for preprocessing and analysis of the fMRI data, implemented in MATLAB 6 (Mathworks). All volumes were temporally realigned to the acquisition of the middle slice and were spatially realigned to the first volume of the first time series. Resulting volumes were normalized to a standard EPI template based on the Montreal Neurological Institute (MNI) reference brain (Cocosco et al. 1997) in Talairach space and resampled to $3 \mathrm{~mm}^{3}$ voxels using nonlinear basis functions (Ashburner and Friston 1999). Normalized images were smoothed with an $8 \mathrm{~mm}$ fullwidth half-maximum Gaussian kernel. The time series in each voxel was high-pass filtered to $1 / 128 \mathrm{~Hz}$ to remove low-frequency noise and scaled within session to a grand mean of 100 across both voxels and scans. Each subject's T1-weighted anatomical scan was normalized to a standard T1 template of the MNI brain. After normalization, an across-subject mean T1-weighted anatomical scan was created. 
Statistical analysis was performed on the study phase data using a two-stage mixed effects model. In the first stage, neural activity was modeled by delta functions (impulse events) at stimulus onset. Then, the ensuing BOLD response was modeled by convolving these neural functions with a canonical hemodynamic response function (HRF) and its temporal and dispersion derivatives (Friston et al. 1998). In the main analysis of subsequent source memory effects, two events of interest were defined for each study task: source correct (correctly recognized items accorded accurate source judgments) and source incorrect (correctly recognized items accorded inaccurate or unsure source judgments). Missed items (study items incorrectly endorsed as new) and words given incorrect responses or no response at the time of study were modeled as events of no interest, along with buffer items. Also included in the analysis model were six covariates modeling movement-related residual variance (the three rigid body translations and three rotations determined from the realignment stage) and two session-specific terms modeling the mean over scans in each session. For the subsidiary analysis of subsequent item memory effects, the relevant events of interest were source incorrect items (defined as above) and misses. Parameter estimates for events of interest were estimated using a general linear model. Nonsphericity of the error covariance was accommodated by an AR(1) model, in which the temporal autocorrelation was estimated by pooling over suprathreshold voxels (Friston et al. 2002). The parameters for each covariate and the hyperparameters governing the error covariance were estimated using restricted maximum likelihood.

In the second stage of the analysis, linear contrasts of the parameter estimates were performed for each subject, treating subjects as a random effect. Separate contrasts were performed with parameter estimates derived from the canonical HRF and each of the two derivatives. The results from the temporal and dispersion derivatives did not substantially add to the results obtained from the canonical HRF, and are not reported. Unless otherwise specified, clusters identified by contrasts between the activity elicited by the different classes of study item were considered reliable when they met the combination of a peak threshold of $p<0.005$ and a cluster extent threshold of $p<0.05$ (approximating to 36 voxels in the primary analyses).

\section{Acknowledgments}

This research was supported by National Institute of Mental Health Grant MH074528. We thank the members of the University of California-Irvine Research Imaging Center for their assistance with fMRI data acquisition.

\section{References}

Ashburner, J. and Friston, K. 1999. Nonlinear spatial normalization using basis functions. Hum. Brain Mapp. 7: 254-266.

Bartels, A. and Zeki, S. 2000. The architecture of the colour centre in the human visual brain: New results and a review. Eur. J. Neurosci. 12: $172-193$.

Bornstein, B.H. and LeCompte, D.C. 1995. A comparison of item and source forgetting. Psychon. Bull. Rev. 2: 254-259.

Brewer, J.B., Zhao, Z., Desmond, J.E., Glover, G.H., and Gabrieli, J.D.E. 1998. Making memories: Brain activity that predicts how well visual experience will be remembered. Science 281: 1185-1187.

Cansino, S., Maquet, P., Dolan, R.J., and Rugg, M.D. 2002. Brain activity underlying encoding and retrieval of source memory. Cereb. Cortex 12: $1048-1056$.

Cocosco, C.A., Kollokian, V., Kwan, R.S., and Evans, A.C. 1997. Brainweb: Online interface to a 3D MRI simulated brain database. In Proceedings of the $3^{\text {rd }}$ International Conference on Functional Mapping of the Human Brain, p. S245. Copenhagen, Denmark.

Davachi, L. 2006. Item, context and relational episodic encoding in humans. Curr. Opin. Neurobiol. 16: 693-700.

Davachi, L., Mitchell, J.P., and Wagner, A.D. 2003. Multiple routes to memory: Distinct medial temporal lobe processes build item and source memories. Proc. Natl. Acad. Sci. 100: 2157-2162.

Eichenbaum, H., Yonelinas, A.R., and Ranganath, C. 2007. The medial temporal lobe and recognition memory. Annu. Rev. Neurosci. 30: $123-152$.

Fletcher, P.C., Stephenson, C.M., Carpenter, T.A., Donovan, T., and Bullmore, E.T. 2003. Regional brain activations predicting subsequent memory success: An event-related fMRI study of the influence of encoding tasks. Cortex 39: 1009-1026.

Friston, K.J., Fletcher, P., Josephs, O., Holmes, A., Rugg, M.D., and Turner, R. 1998. Event-related fMRI: Characterizing differential responses. Neuroimage 7: 30-40.

Friston, K.J., Penny, W., Phillips, C., Kiebel, S., Hinton, G., and Ashburner, J. 2002. Classical and Bayesian inference in neuroimaging: Applications. Neuroimage 16: 484-512.

Glanzer, M., Hilford, A., and Kim, K. 2004. Six regularities of source recognition. J. Exp. Psychol. 30: 1176-1195.

Gold, J.J., Smith, C.N., Bayley, P., Shrager, Y., Brewer, J., Stark, C.E.L., Hopkins, R., and Squire, L.R. 2006. Item memory, source memory, and the medial temporal lobe: Concordant findings from fMRI and memory-impaired patients. Proc. Natl. Acad. Sci. 103: 9351-9356.

Grill-Spector, K., Kourtzi, Z., and Kanwisher, N. 2001. The lateral occipital complex and its role in object recognition. Vision Res. 41: $1409-1422$

Habib, R., Nyberg, L., and Tulving, E. 2003. Hemispheric asymmetries of memory: The HERA model revisited. Trends Cogn. Sci. 7: 241-245.

Hayman, C. and Rickards, C. 1995. A dissociation in the effects of study modality on tests of implicit and explicit memory. Mem. Cognit. 23: $95-112$.

Henson, R.N.A., Rugg, M.D., Shallice, T., Josephs, O., and Dolan, R.J. 1999. Recollection and familiarity in recognition memory: An event-related fMRI study. J. Neurosci. 19: 3962-3972.

Johnson, M.K., Hashtroudi, S., and Lindsay, D.S. 1993. Source monitoring. Psychol. Bull. 114: 3-28.

Kensinger, E.A. and Schacter, D.L. 2006. Amygdala activity is associated with the successful encoding of item, but not source, information for positive and negative stimuli. J. Neurosci. 26: 2564-2570.

Kirchhoff, B.A., Wagner, A.D., Maril, A., and Stern, C.E. 2000. Prefrontal-temporal circuitry for episodic encoding and subsequent memory. J. Neurosci. 20: 6173-6180.

Kolers, P.A. 1973. Remembering operations. Mem. Cognit. 1: 347-355.

Kučera, H. and Francis, W.N. 1967. Computational analysis of present-day American English. Brown University Press, Providence, RI.

Lazar, N.A., Luna, B., Sweeney, J.A., and Eddy, W. 2002. Combining brains: A survey method for statistical pooling of information. Neuroimage 16: 538-550.

Lerner, Y., Handler, T., and Malach, R. 2002. Object-completion effects in the human lateral occipital complex. Cereb. Cortex 12: 163-177.

Mitchell, J.P., Macrae, C.N., and Banaji, M.R. 2004. Encoding-specific effects of social cognition on the neural correlates of subsequent memory. J. Neurosci. 24: 4912-4917.

Morcom, A.M., Good, C.D., Frackowiak, R.S., and Rugg, M.D. 2003. Age effects on the neural correlates of successful memory encoding. Brain 126: $213-229$.

Morris, C.D., Bransford, J.D., and Franks, J.J. 1977. Levels of processing versus transfer appropriate processing. J. Verbal Learning Verbal Behavior 16: 519-533.

Naveh-Benjamin, M. and Craik, F.I.M. 1996. Effects of perceptual and conceptual processing on memory for words and voice: Different patterns for young and old. Q. J. Exp. Psychol. A 49: 780-796.

Otten, L.J. 2007. Fragments of a larger while: Retrieval cues constrain observed neural correlates of memory encoding. Cereb. Cortex 17: 2030-2038.

Otten, L.J. and Rugg, M.D. 2001. Task-dependency of the neural correlates of episodic encoding as measured by fMRI. Cereb. Cortex 11: $1150-1160$.

Otten, L.J., Henson, R.N.A., and Rugg, M.D. 2001. Depth of processing effects on neural correlates of memory encoding-Relationship between findings from across- and within-task comparisons. Brain 124: 399-412.

Otten, L.J., Henson, R.N.A., and Rugg, M.D. 2002. State-related and item-related neural correlates of successful memory encoding. Nat. Neurosci. 5: 1339-1344.

Paller, K.A. and Wagner, A.D. 2002. Observing the transformation of experience into memory. Trends Cogn. Sci. 6: 93-102.

Park, H. and Rugg, M.D. 2008. The relationship between study processing and the effects of cue congruency at retrieval: fMRI support for transfer appropriate processing. Cereb. Cortex 18: $868-875$.

Ranganath, C., Yonelinas, A.P., Cohen, M.X., Dy, C.J., Tom, S., and D'Esposito, M. 2004. Dissociable correlates for familiarity and recollection within the medial temporal lobes. Neuropsychologia 42: $2-13$.

Roediger, H.L., Gallo, D.A., and Geraci, L. 2002. Processing approaches to cognition: The impetus from the levels-of-processing framework. Memory 10: 319-332.

Rugg, M.D., Otten, L.J., and Henson, R.N. 2002. The neural basis of episodic memory: Evidence from functional neuroimaging. Philos. Trans. R. Soc. London, Ser. B 357: 1097-1110. 
Rugg, M.D., Johnson, J.D., Park, H., and Uncapher, M.R. 2008. Encoding-retrieval overlap in human episodic memory: A functional neuroimaging perspective. In The essence of memory, 169 (eds. W. Sossin et al.), Section IV. Elsevier, Amsterdam.

Smith, S.M., Beckmann, C.F., Ramnani, N., Woolrich, M.W., Bannister, P.R., Jenkinson, M., Matthews, P.M., and McGonigle, D.J. 2005. Variability in fMRI: A re-examination of inter-session differences. Hum. Brain Mapp. 24: 248-257.

Snodgrass, J.G. and Corwin, J. 1988. Pragmatics of measures recognition memory: Applications to dementia and amnesia. J. Exp. Psychol. 117: $34-50$.

Tendolkar, I., Arnold, J., Petersson, K.M., Weis, S., Brockhaus-Dumke, A., Van Eijndhoven, P., Buitelaar, J., and Ferandez, G. 2007. Probing the neural correlates of associative memory formation: A parametrically analyzed event-related functional MRI study. Brain Res. 1142: $159-168$.

Uncapher, M.R. and Rugg, M.D. 2005. Encoding and the durability of episodic memory: A functional magnetic resonance imaging study. $J$.
Neurosci. 25: 7260-7267.

Uncapher, M.R., Otten, L.J., and Rugg, M.D. 2006. Episodic encoding is more than the sum of its parts: An fMRI investigation of multifeatural contextual encoding. Neuron 52: 547-556.

Wagner, A.D., Schacter, D.L., Rotte, M., Koutstaal, W., Maril, A., Dale, A.M., Rosen, B., and Buckner, R.L. 1998. Building memories: Remembering and forgetting of verbal experiences as predicted by brain activity. Science 281: 1188-1191.

Yonelinas, A.P. 2002. The nature of recollection and familiarity: A review of 30 years of research. J. Mem. Lang. 46: 441-517.

Yonelinas, A.P. and Jacoby, L.L. 1996. Noncritical recollection: Familiarity as automatic, irrelevant recollection. Conscious. Cogn. 5: $131-141$.

Zeki, S. and Marini, L. 1998. Three cortical stages of colour processing in the human brain. Brain 121: 1669-1685.

Received December 10, 2007; accepted in revised form April 10, 2008. 
Learning \& Memory 15: 143-152 (2008)

\section{Activation of CB1 specifically located on GABAergic interneurons inhibits LTD in the lateral amygdala}

Shahnaz Christina Azad, Jörg Kurz, Giovanni Marsicano, Beat Lutz, Walter Zieglgänsberger, and Gerhard Rammes

One sentence was inadvertently omitted from the Acknowledgments section:

G.M. was supported by AVENIR INSERM, in partnership with the Bettencourt-Schueller Foundation. S.C.A. and G.R. were supported by a grant from the Deutsche Forschungsgemeinschaft (DFG; AZ 71/1-1 and RA689/7-1).

Learning \& Memory 15: 417-425 (2008)

Effects of study task on the neural correlates of source encoding Heekyeong Park, Melina R. Uncapher, and Michael D. Rugg

For the reference Rugg et al. 2008, the book title was incorrectly cited. The correct book title is Progress in brain research. The corrected reference should read:

Rugg, M.D., Johnson, J.D., Park, H., and Uncapher, M.R. 2008. Encoding-retrieval overlap in human episodic memory: A functional neuroimaging perspective. In Progress in brain research, 169 (eds. W. Sossin et al.), Section IV. Elsevier, Amsterdam. 


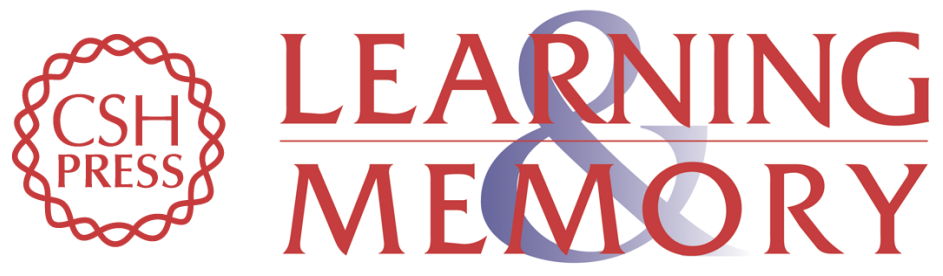

\section{Effects of study task on the neural correlates of source encoding}

Heekyeong Park, Melina R. Uncapher and Michael D. Rugg

Learn. Mem. 2008, 15:

Access the most recent version at doi:10.1101//m.878908

\section{Related Content Errata}

Learn. Mem. July , 2008 15: 550

References This article cites 45 articles, 9 of which can be accessed free at: http://learnmem.cshlp.org/content/15/6/417.full.html\#ref-list-1

Articles cited in:

http://learnmem.cshlp.org/content/15/6/417.full.html\#related-urls

\section{License}

Email Alerting Receive free email alerts when new articles cite this article - sign up in the box at the Service top right corner of the article or click here. 